\title{
Knowledgeable Feedback via a Cast of Virtual Characters with Different Competences
}

\author{
Wouter Beek ${ }^{1}$, Jochem Liem ${ }^{1}$, Floris Linnebank ${ }^{1}$, René Bühling ${ }^{2}$, Michael Wißner ${ }^{2}$, \\ Esther Lozano ${ }^{3}$, Jorge Gracia del Río ${ }^{3}$, and Bert Bredeweg ${ }^{1}$ \\ ${ }^{1}$ University of Amsterdam, Informatics Institute, Amsterdam, Netherlands \\ ${ }^{2}$ University of Augsburg, Multimedia Concepts and Applications, Augsburg, Germany \\ ${ }^{3}$ Universidad Politécnica de Madrid, Ontology Engineering Group, Madrid, Spain
}

\begin{abstract}
DynaLearn (http://www.DynaLearn.eu) develops a cognitive artefact that engages learners in an active learning by modelling process to develop conceptual system knowledge. Learners create external representations using diagrams. The diagrams capture conceptual knowledge using the Garp3 Qualitative Reasoning (QR) formalism [2]. The expressions can be simulated, confronting learners with the logical consequences thereof. To further aid learners, DynaLearn employs a sequence of knowledge representations (Learning Spaces, LS), with increasing complexity in terms of the modelling ingredients a learner can use [1]. An online repository contains QR models created by experts/teachers and learners. The server runs semantic services [4] to generate feedback at the request of learners via the workbench. The feedback is communicated to the learner via a set of virtual characters, each having its own competence [3]. A specific feedback thus incorporates three aspects: content, character appearance, and a didactic setting (e.g. Quiz mode). In the interactive event we will demonstrate the latest achievements of the DynaLearn project. First, the 6 learning spaces for learners to work with. Second, the generation of feedback relevant to the individual needs of a learner using Semantic Web technology. Third, the verbalization of the feedback via different animated virtual characters, notably: Basic help, Critic, Recommender, Quizmaster \& Teachable agent.
\end{abstract}

\section{References}

1. Bredeweg, B., Liem, J., Beek, W., Salles, P., Linnebank, F.: Learning spaces as representational scaffolds for learning conceptual knowledge of system behaviour. In: Wolpers, M., Kirschner, P.A., Scheffel, M., Lindstaedt, S., Dimitrova, V. (eds.) EC-TEL 2010. LNCS, vol. 6383, pp. 46-61. Springer, Heidelberg (2010)

2. Bredeweg, B., Linnebank, F.E., Bouwer, A.J., Liem, J.: Garp3 - Workbench for qualitative modelling and simulation. Ecological Informatics 4(5-6), 263-281 (2009)

3. Mehlmann, G., Häring, M., Bühling, R., Wißner, M., André, E.: Multiple agent roles in an adaptive virtual classroom environment. In: Proc. of the 10th Int. Conf. on Intelligent Virtual Agents, pp. 250-256. Springer, Heidelberg (2010)

4. Gracia, J., Liem, J., Lozano, E., Corcho, O., Trna, M., Gómez-Pérez, A., Bredeweg, B.: Semantic techniques for enabling knowledge reuse in conceptual modelling. In: PatelSchneider, P.F., Pan, Y., Hitzler, P., Mika, P., Zhang, L., Pan, J.Z., Horrocks, I., Glimm, B. (eds.) ISWC 2010, Part II. LNCS, vol. 6497, pp. 82-97. Springer, Heidelberg (2010) 